Charles Mesguich*

Krimo Bouabdallah

Laurence Bordenave

Noël Milpied

Elif Hindié

*CHU Bordeaux

Avenue de Magellan

Pessac, France 33604

E-mail: charles.mesguich@chu-bordeaux.fr
Published online Feb. 14, 2020.

DOI: 10.2967/jnumed.120.242594

\section{Reply: Interim PET Assessment of Advanced Hodgkin Lymphoma: Is It Sufficient?}

REPLY: Mesguich et al. (1) commented on our publication, which reported the long-term outcome of patients with advancedstage Hodgkin lymphoma undergoing interim ${ }^{18} \mathrm{~F}$-FDG PET (after 2 cycles of chemotherapy; PET2) and PET2 response-adapted change in therapy in the SWOG S0816 trial $(2,3)$. Their letter emphasizes the importance of the end-of-treatment PET (PET3). Of the 270 patients with a negative PET2 scan result from the S0816 study, 244 patients (90\%) underwent PET3 and 35 of these 244 patients (14\%) had a positive PET3 scan result (3). Unfortunately, our study did not mandate biopsy after positive PET3, so we are not able to confirm that all 35 of these patients actually had residual disease. At the time of positive PET3, 13 of the 35 patients were officially categorized as disease progression. Nineteen of the 35 patients initiated salvage therapy shortly after the PET3 scan. Therefore, it remains possible that some of the positive PET3 scans represent a false-positive result. However, we agree with the assessment that the PET3 scan can be a useful tool to identify pri- mary refractory patients requiring expedited salvage therapy. Nevertheless, PET2 remains very important in the early detection of highrisk patients treated with standard chemotherapy regimens, who are unlikely to show a complete response to therapy, which may justify intensification of treatment at that time, contributing to improved survival (2). In addition, we note that when novel targeted agents such as brentuximab vedotin and nivolumab are used in combination with standard chemotherapy agents, PET2-based responses are not used to alter therapy. As such, the ongoing national SWOG S1826 study compares brentuximab vedotin or nivolumab in combination with adriamycin, vinblastine, and dacarbazine and does not modify therapy based on the PET2 scan results (NCT03907488).

\section{REFERENCES}

1. Mesguich C, Bouabdallah K, Bordenave L, Milpied N, Hindié E. Interim PET assessment of advanced Hodgkin lymphoma: is it sufficient? J Nucl Med. 2020; 61:1694-1695.

2. Press OW, Li H, Schoder H, et al. US intergroup trial of response-adapted therapy for stage III to IV Hodgkin lymphoma using early interim fluorodeoxyglucosepositron emission tomography imaging: Southwest Oncology Group S0816. J Clin Oncol. 2016;34:2020-2027.

3. Stephens DM, Li H, Schöder H, et al. Five-year follow-up of SWOG S0816: limitations and values of a PET-adapted approach with stage III/IV Hodgkin lymphoma. Blood. 2019;134:1238-1246.

Deborah M. Stephens* Heiko Schoder Hongli Li Jonathan Friedberg 1950 Circle of Hope, RS5509 University of Utah Salt Lake City, UT 84112 E-mail: deborah.stephens@hci.utah.edu

Published online Sep. 11, 2020.

DOI: 10.2967/jnumed.120.242685 